VERSITA

\author{
TATRA \\ MOUNTaiNs \\ Mathematical Publications \\ DOI: $10.2478 / \mathrm{v} 10127-011-0028-y$ \\ Tatra Mt. Math. Publ. 49 (2011), 89-98
}

\title{
FUNCTIONS WITH BOUNDED VARIATION IN LOCALLY CONVEX SPACE
}

\author{
Miloslav Duchoñ — Camille Debiève
}

\begin{abstract}
The present paper is concerned with some properties of functions with values in locally convex vector space, namely functions having bounded variation and generalizations of some theorems for functions with values in locally convex vector spaces replacing Banach spaces, namely Theorem: If $X$ is a sequentially complete locally convex vector space, then the function $x(\cdot):[a, b] \rightarrow X$ having a bounded variation on the interval $[a, b]$ defines a vector-valued measure $m$ on borelian subsets of $[a, b]$ with values in $X$ and with the bounded variation on the borelian subsets of $[a, b]$; the range of this measure is also a weakly relatively compact set in $X$. This theorem is an extension of the results from Banach spaces to locally convex spaces.
\end{abstract}

\section{Introduction}

The set $\mathcal{B V}$ of functions $x(\cdot)$ of finite (locally bounded) variation, on the real line $R$ to a complete complex locally convex vector space $X$ derives its importance from the fact that each function $x(\cdot)$ in $\mathcal{B V}$ is associated in a natural way with a vector-valued measure on the $\operatorname{ring} \mathcal{B}$ of bounded Borel sets, and conversely. The relationship resembles that between ordinary real and complex functions of bounded variation and real and signed measures defined on $\mathcal{B}$. A precise statement will be given later in Section 4.

As for some older results, see ([1], [2], [4], [5], [7], [8], [10], [13]). The extension to locally convex vector space (shortly lcs) is very useful because it allows to consider many important spaces, which are not normable, e.g., nuclear locally convex vector spaces often appearing in applications (see [9]). The paper

(C) 2011 Mathematical Institute, Slovak Academy of Sciences.

2010 Mathematics Subject Classification: 28E10, 81P10.

Keywords: locally convex space, bounded variation, vector-valued measure on Borel subsets.

Supported by grant agency VEGA, no. 2/0212/10. 


\section{MILOSLAV DUCHOŇ — CAMILLE DEBIÈVE}

is divided into four sections. Most propositions and theorems are proved under the assumption that lcs $X$ is a sequentially complete (further called $s$-complete) Hausdorff locally convex topological vector space.

\section{Functions with bounded variation in lcs}

In the second section there are investigated functions $x(\cdot):[a, b] \rightarrow X$ with the bounded $p$-variation, where $X$ is lcs. Let $P$ be a set of seminorms determining the topology of $X$.

Definition 1. A function $x(\cdot):[a, b] \rightarrow X$ has a bounded $p$-variation on $[a, b]$, if for arbitrary semi-norm $p \in P$ there exists a real number $K_{p}(a, b), 0 \leq$ $K_{p}(a, b)<\infty$ such that for any $k \geq 1$ and an arbitrary division of the interval $[a, b]$ such that

$$
-\infty<a \leq t_{0}<t_{1}<\cdots<t_{k} \leq b<\infty
$$

it is

$$
\sum_{s=1}^{k} p\left[x\left(t_{s}\right)-x\left(t_{s-1}\right)\right] \leq K_{p}(a, b) .
$$

The smallest of the numbers $K_{p}(a, b)$ we shall call the $p$-variation of the function $x(\cdot)$ on $[a, b]$ and denote $p_{v}[x(\cdot),[a, b]]$.

For definition of bounded variation in Banach spaces see [6].

From the definition we obtain the following.

Proposition 1. If $x(\cdot):[a, b] \rightarrow X$ has a bounded $p$-variation on $[a, b]$, then the scalar-valued function $x^{\prime} x(\cdot)$ has the bounded variation on $[a, b]$ for any $x^{\prime} \in X^{\prime}$.

P r o o f. It suffices for $p$ to take $\left|\left(., x^{\prime}\right)\right|$.

Hence we easily the following.

Proposition 2. If the function $x(\cdot):[a, b] \rightarrow X$ has the bounded p-variation on $[a, b]$, then the scalar-valued function $x^{\prime} x$ can be, for any $x^{\prime} \in X^{\prime}$, expressed in the form

$$
x^{\prime} x(t)=\sum_{r=0}^{3} i^{r} f_{r}\left(t, x^{\prime}\right), \quad t \in[a, b],
$$

where $f_{r}\left(t, x^{\prime}\right), r=0,1,2,3$, are finite real-valued nondecreasing functions of variable $t$.

Note that the functions $f_{r}$ are not uniquely determined. Recall the following definitions. 
Definition 2. The series $\sum x_{i}, x_{i} \in X$, unconditionally (permutally) converges, if for any permutation $p(i)$ of natural numbers the series $\sum x_{p(i)}$ converges in $X$.

Definition 3. The series $\sum x_{i}, x_{i} \in X$, subseries converges, if for any increasing sequence $\left(n_{i}\right)$ of natural numbers the series $\sum x_{n_{i}}$ converges to some element in $X$.

Note that in $s$-complete lcs the unconditional and subseries convergence coincide.

The following results are generalizations, proved in detail in [11] for lcs, of the results proved in [5] for normed spaces.

Proposition 3. Let $X$ be s-complete lcs and let $x(\cdot):[a, b] \rightarrow X$ have the bounded p-variation on $[a, b]$. Denote

$$
\begin{aligned}
& u(t)=x(t)-x(t-0), \\
& v(t)=x(t+0)-x(t)
\end{aligned}
$$

and let $\left(t_{n}\right)$ be any sequence of different values belonging to the interior of the compact interval $[a, b]$. Then the series

$$
\sum_{n=1}^{\infty} u\left(t_{n}\right), \quad \sum_{n=1}^{\infty} v\left(t_{n}\right)
$$

converge absolutely, hence unconditionally converge in the space $X$.

For metrisable spaces we have the following result.

Proposition 4. If $X$ is a complete metrisable lcs and $x(\cdot): R \rightarrow X$ has on every interval $[a, b]$ the bounded $p$-variation and $u(t), v(t)$ are the functions defined in Proposition 3, then

$$
u(t)=v(t)=0
$$

except for at most countable many $t$.

We can conclude the preceding propositions into

THEOREM 4. If $X$ is an s-complete lcs and $x(\cdot): R \rightarrow X$ has on every interval $[a, b]$ the bounded p-variation, then the limits of the function $x(\cdot)$ from the left, and from the right, $x(t \pm 0) \in X$, exist in every point $t$ and also there exist $\lim _{t \rightarrow \infty} x(t)$ and $\lim _{t \rightarrow-\infty} x(t)$ if $a=-\infty, b=\infty$.

For metrisable spaces we have

TheOrem 5. If $X$ is a complete metrisable lcs and $x(\cdot): R \rightarrow X$ has on every interval $[a, b]$ the bounded p-variation, then the function $x(\cdot)$ is continuous for all $t$ except of at most of the countable set. 
Theorem 6. If $X$ is a s-complete lcs and $x(\cdot):[a, b] \rightarrow X$ has on $[a, b]$ the bounded $p$-variation, then the function defined by the relation

$$
\tilde{x}(t)=x(t+0), \quad t \in[a, b],
$$

has the bounded p-variation on $[a, b]$.

We now give more results for metrisable lcs.

Proposition 5. Let $X$ be a complete metrisable lcs and let $s_{n}^{p}, n=1,2, \ldots, p \in P$ be all points of discontinuity of the function $x(\cdot): R \rightarrow X$ continuous from the right and with bounded p-variation on every compact interval $[a, b]$. Then we can define the step function $z(\cdot)$ and the function $w(\cdot)$ with

$$
w(t)=x(t)-z(t)
$$

such that the functions $z(\cdot)$ and $w(\cdot)$ have the bounded p-variation on every compact interval $[a, b]$ and in all points $t$ there exist limits of the functions $w(\cdot)$ and $z(\cdot)$ from the left and from the right, $z(t \pm 0)$ and $w(t \pm 0)$. Moreover it is

$$
w(t-0)=w(t)=w(t+0) .
$$

We can formulate the obtained results in the form of the following main theorems.

THEOREM 7. If $X$ is a complete metrisable lcs and $x(\cdot): R \rightarrow X$ has the bounded $p$-variation on every compact interval $[a, b]$ and is everywhere continuous from the right, then

$$
x(t)=z(t)+w(t), \quad t \in(-\infty, \infty),
$$

where the functions $z(\cdot)$ and $w(\cdot)$ have the bounded p-variation on every compact interval $[a, b], w(\cdot)$ is everywhere continuous and $z(\cdot)$ is stepwise function from the Proposition 5.

TheOREM 8. If $X$ is a complete metrisable lcs and $x(\cdot): R \rightarrow X$ has the bounded p-variation on every compact interval $[a, b]$, then $x(\cdot)$ has a separable set of values.

P r o o f. Consider $A=\{x(t), t \in Q\}$, where $Q$ is the set of all rational numbers. Then $A \subset H$, where $H$ is the set of values of the function $x(\cdot)$. The function $x(\cdot+0)$ differs from $x(\cdot)$ at most in countably many points. Denote $B=\{x(t), x(t) \neq$ $x(t \pm 0)\}$. Then $A \cup B$ is a countable set, $A \cup B \subset H$, and simultaneously $H \subset \overline{A \cup B}$, i.e., the set $H$ contains a countable dense subset and hence is separable. 


\section{FUNCTIONS WITH BOUNDED VARIATION IN LOCALLY CONVEX SPACE}

\section{Connection with vector-valued measures}

Now we shall try to derive, given the function with the bounded $p$-variation, a corresponding borelian measure $m$ on the $\delta$-ring of borelian subsets, with values in $X$, which has the bounded $p$-variation on every compact interval $[a, b]$,

$$
|m|_{p}(A)=\sup \sum_{k}\left|m\left(A_{k}\right)\right|_{p}
$$

where the supremum is taken over all finite families $\left(A_{k}\right)$ of mutually disjoint Borel subsets of $A$. It follows that $|m|_{p}$ is the smallest Borel measure $\mu_{p}^{m}$ such that

$$
|m(A)|_{p} \leq \mu_{p}^{m}(A)
$$

for all Borel sets $A$.

In this section, there is investigated the existence of the unique correspondence between the vector valued functions with $p$-bounded variation and continuous from the right on $[a, b]$ and the vector valued measures on borelian sets in $[a, b]$, with values in $X$, and with $p$-bounded variation.

Denote by $\mathcal{B}[a, b]$ the $\sigma$-algebra of borelian subsets of the interval $[a, b]$. Let $\mathcal{B}$ be the union of all $\sigma$-algebras $\mathcal{B}[a, b], a, b \in R$. Then $\mathcal{B}$ is the $\delta$-ring. Recall that a $\delta$-ring $\mathcal{S}$ is a ring having the property: for every sequence $\left(A_{i}\right)$ of sets of $\mathcal{S}$ there holds $\bigcap_{i=1}^{\infty} A_{i} \in \mathcal{S}$.

Definition 9. A function $m: \mathcal{B} \rightarrow X$, where $X$ is lcs and $\mathcal{B}$ is the $\delta$-ring of borelian subsets of the interval $[a, b]$, is called vector valued measure, if

$$
\begin{aligned}
m(\emptyset) & =0, \\
m\left(\bigcup_{n=1}^{\infty} E_{n}\right) & =\sum_{n=1}^{\infty} m\left(E_{n}\right),
\end{aligned}
$$

if the sets $E_{n} \in \mathcal{B}$ are mutually disjoint, with the union in $\mathcal{B}$, the series in the right being unconditionally convergent.

TheOREM 10. If $X$ is a s-complete lcs and $m: \mathcal{B} \rightarrow X$ is a vector-valued measure, with the bounded p-variation, then the function $x(\cdot): R \rightarrow X$ defined by the relations

$$
\begin{gathered}
x(0)=0, \\
x(d)-x(c)=m(E), \quad E=(c, d), c, d \in R,-\infty<c<d<\infty,
\end{gathered}
$$

has the bounded p-variation on every compact interval $[a, b]$ and is everywhere strongly continuous from the right. 
Pr o of. Since $m$ is vector-valued measure, with the finite $p$-variation, for any compact interval $[a, b]$ the set

$$
\begin{aligned}
& E(a, b)_{p} \\
& =\left\{\sum_{i=1}^{M} p\left[x\left(t_{2 i}\right)-x\left(t_{2 i-1}\right)\right], M \geq 1,-\infty<a \leq t_{1}<t_{2}<\cdots<t_{2 n} \leq b<\infty .\right. \\
& \left.=\sum_{i=1}^{M} p\left(m\left(E_{i}\right)\right), \text { with } E_{i}=\left(t_{2 i-1}, t_{2 i}\right] \text { being disjoint }\right\},
\end{aligned}
$$

is $p$-bounded, for any interval $[a, b]$, i.e., the function $x(\cdot)$ has the bounded $p$-variation for any interval $[a, b]$, and it is easy to prove that it is continuous from the right.

Conversely we have the following.

TheOrem 11. If $X$ is a s-complete lcs and $x(\cdot): R \rightarrow X$ has the bounded $p$-variation on every interval $[a, b]$ and is continuous from the right, then there exists a unique vector-valued measure $m: \mathcal{B} \rightarrow X$ with the bounded p-variation, such that

$$
m(E)=x(d)-x(c), \quad E=(c, d) .
$$

P r o of. Let $x(\cdot)$ be a function with the bounded $p$-variation on every compact interval $[a, b]$ and let it be continuous from the right. Let $m$ be defined by the relation

$$
m(E)=x(d)-x(c), \quad E=(c, d),
$$

Denote by $\mathcal{G}$ the class of the intervals of the form $(a, b]$. It is easy verified that $\mathcal{G}$ is a semi-ring. We shall denote by $\mathcal{C}$ the ring generated by $\mathcal{G}$. The ring $\mathcal{C}$ consists of all finite unions of disjoint intervals of $\mathcal{G}$. Every non void set $A$ of $\mathcal{C}$ can be written as a finite union $A=\bigcup_{i=1}^{n} I_{i}$ of disjoint non void intervals of $\mathcal{G}$ such that any two intervals $I_{i}$ and $I_{j}$ have in common no extremity.

We remind that an additive set function $m: \mathcal{G} \rightarrow X$ can be uniquely extended to an additive set function (denoted again) $m: \mathcal{C} \rightarrow X$ and that the variations of $m$ and its extension are equal. Further $m: \mathcal{C} \rightarrow X$ can be uniquely extended to a $\sigma$-additive set function with the finite variation, $m: \mathcal{B} \rightarrow X$. The variation of the extension of $m$ from $\mathcal{G}$ to $\mathcal{B}$ is equal to the unique extension of $\mu^{m}$ from $\mathcal{G}$ to $\mathcal{B}$. The details (rather long even for Banach space) can be compared in ([3]).

\section{Functions and measures}

In this section we shall show more concretely that the Borel measure of finite variation can be identified with the function of finite variation. 


\section{FUNCTIONS WITH BOUNDED VARIATION IN LOCALLY CONVEX SPACE}

Now consider the set $\mathcal{B} \mathcal{V}_{0}$ of functions $x(\cdot)$ of finite-locally bounded-variation and continuous from the right, on the real line $R$ to a complete complex locally convex vector space $X$. It derives its importance from the fact that each function $x(\cdot)$ in $\mathcal{B} \mathcal{V}_{0}$ is associated in a natural way with a vector-valued measure on the ring $\mathcal{B}$ of bounded Borel sets, and conversely.

If the function $x(\cdot) \in \mathcal{B} \mathcal{V}_{0}$ is given, and the associated $m: \mathcal{B} \cap[a, b] \rightarrow X$ and $y:[a, b] \rightarrow X, y(t)=m([a, t]),(a<t \leq b), y(a)=0$, then $y(\cdot) \in \mathcal{B} \mathcal{V}$. By standard argument

$$
x(t)-x(a)=y(t)-y(a)(a \leq t \leq b) .
$$

Hence $y(a)=y(a+0)$ and therefore $m((a))=0$, so that

$$
y(t)=m([a, t])=m([a, t])-m((a))=m((a, t]) \quad(a<t \leq b) .
$$

If we denote by $m_{0}$ the restriction of $m \rightarrow \mathcal{B} \cap(a, b]$, then we have

$$
m_{0}(E)=y(d)-y(c)=x(d)-x(c)
$$

whenever $E=(c, d] \subset(a, b]$.

We can then construct a vector valued measure $m_{0}$ for $-\infty<a<b<\infty$ and it will be possible to complete the proof of the following

Corollary 12. If $x(\cdot): R \rightarrow X$ is a function of the class $\mathcal{B} \mathcal{V}_{0}$, where $X$ is an arbitrary s-complete lcs, then there exists a unique vector-valued measure $m: \mathcal{B} \rightarrow X$ of bounded variation satisfying

$$
x(d)-x(c)=m(E), \quad E=(c, d) .
$$

Remark 13. It is possible to prove that it suffices to know the values of $m$ only for the intervals of the form $(a, b)$ with $a<b$, or only for the intervals $[a, b]$, or only for the intervals $(a, b]$, because each of these classes generates the $\delta$-ring $\mathcal{B}$ $([3])$.

THEOREM 14. If $X$ is an s-complete lcs and the function $x(\cdot):[a, b] \rightarrow X$ has the bounded p-variation on every $[a, b]$ and is continuous from the right, then the vector valued measure $m: \mathcal{B} \rightarrow X$ derived from $x(\cdot)$ by the relation

$$
m(E)=x(d)-x(c),
$$

if $E=(c, d)$, has for every $[a, b]$ the finite $p$-variation with respect to $\mathcal{B}[a, b]$.

Pr o of. Let $p \in P$ be an arbitrary seminorm. For any interval $[a, b]$ we put

$$
v_{p}(t)=v_{p}[x(\cdot),[a, t]], \quad t \in[a, b] .
$$


The function $v_{p}(\cdot)$ is finite, nondecreasing and nonnegative scalar function such that for any pair $s, t \in[a, b], s<t$ there holds

$$
\begin{aligned}
p[x(t)-x(s)] & \leq v_{p}[x(\cdot),[s, t]] \\
& =v_{p}[x(\cdot),[a, t]]-v_{p}[x(\cdot),[a, s]] \\
& =v_{p}(t)-v_{p}(s) .
\end{aligned}
$$

From the properties of the function $v_{p}(\cdot)$ it follows that it has on the fix $[a, b]$ the bounded variation, hence $x(\cdot)$ has the bounded $p$-variation.

Remark 15. Let $T=[a, b]$ and let $g$ be a function of bounded variation ( $[3$, p. 362]). We can always suppose that $g$ is continuous on the right in all points of $T$ except for $b$. In this case, if $u<b$ we have

$$
m((a, u])=g(u)-g(a) .
$$

Then $m$ can be uniquely extended to a regular Borel measure with the bounded variation, defined on the sigma ring of Borel sets $A \subset T=[a, b]$.

Let $g: T \rightarrow X$ be a function with the bounded variation on $T=[a, b]$ and $v(t)=V_{a}^{t}(g)$ be the variation of $g$. If $m: \mathcal{B}([a, b]) \rightarrow X$ is the measure corresponding to $g$ and $\mu$ is the measure corresponding to $v$, and if $g$ is continuous on the right in $(a, b]$ then $\mu$ is the variation of $m$, and if $\mathcal{S}$ is the semiring of the intervals of the form $(u, v]$ and $[u, b]$, then for all $I \in \mathcal{S}$ we have $|m|(I)=\mu(I)$.

Concluding it is quite natural to put the following question: Why we transit from point functions to set functions and conversely. First we note that in praxis, physical, chemical and so on, we consider quantities of two kinds: the states, i.e., intensive quantities or densities, modelled by point functions, called the derived ones, or amounts or the extensive quantities, modelled by set functions, considered in general as the primary quantities. Therefore, mathematical models constructed in terms of measures and integrals have conceptual and often also technical priority with respect to ones constructed in terms of derivatives or point functions.

For a general locally convex space valued measure $m,|m|_{p}$ need not be finite, as shows the following example (see Thom as [12]).

EXAMPle 1 . Let $m=\left(\mu_{n}\right)_{n \in N}$ be a measure with values in $l^{1}(N)$. Then the variation of $m$ with respect to the norm is given by $|m|(A)=\sum_{n}\left|\mu_{n}\right|(A)$. This can be seen by a direct argument but the following proof illustrates some interesting idea.

Suppose that each $\mu_{n}$ is absolutely continuous with respect a measure $\nu$, so $\mu_{n}=f_{n} . \nu$. Then $m$ has the density $f=\left(f_{n}\right)_{n \in N}$ with values in $C^{N}$. The function

$$
x \rightarrow|x|=\sum_{n \in N}\left|x_{n}\right|
$$




\section{FUNCTIONS WITH BOUNDED VARIATION IN LOCALLY CONVEX SPACE}

is a lower semi-continuous generalized semi-norm in $C^{N}$. Hence

$$
\begin{aligned}
|m|(A)=\int_{A}|f(t)| d \nu & =\int_{A} \sum_{n}\left|f_{n}(t)\right| d \nu(t) \\
& =\sum_{n} \int_{A}\left|f_{n}\right|(t) d \nu(t)=\sum_{n}\left|\mu_{n}\right|(A) .
\end{aligned}
$$

If for instance

$$
\begin{aligned}
T & =[0,2 \pi], \\
f_{n}(t) & =\frac{1}{n} e^{-i n t},
\end{aligned}
$$

and $\nu$ is Lebesgue measure we have

$$
|m|(A)=\sum_{n} \frac{1}{n} \nu(A),
$$

i.e., $|m|(A)=0$ if $\nu(A)=0$ and $|m|(A)=\infty$ if $\nu(A)>0$.

\section{REFERENCES}

[1] BARTLE, R. G.-DUNFORD, N.-SCHWARTZ, J.: Weak compactness and vector measures, Canad. J. Math. 7 (1955), 289-305.

[2] DEBIÈVE, C.: Intégration par rapport à une mesure vectorielle, Ann. Soc. Sci. Brux., Sér. I, 11 (1973), 165-185.

[3] DINCULEANU, N.: Vector Measures, in: Hochschulbücher für Mathematik, Vol. 64, VEB Deutscher Verlag der Wissenschaften, Berlin, 1966.

[4] DUNFORD, N.-SCHWARTZ, J. T.: Linear Operators. Part I. Interscience, New York, USA, 1966.

[5] EDWARDS, D. A.: On the continuity properties of functions satisfying a condition of Sirvint's, Q. J. Math., Oxf. II. Ser. 2 (1957), 58-67.

[6] HILlE, E.: Methods in Classical and Functional Analysis. Adison-Wesley Publ. Comp., Massachusets, USA, 1972.

[7] KLUVÁNEK, I.: To theory of vector valued measures II, Mat.-Fyz. Čas., Slov. Akad. Vied 16 (1966), 76-81.

[8] LEWIS, D. R.: Integration with respect to vector measures, Pac. J. Math. 33 (1970), $157-165$.

[9] ROBERTSON, A. P.-ROBERTSON, W. J.: Topological Vector Spaces. Cambridge Univ. Press, Cambridge, 1964.

[10] SIRVINT, G.: Weak compactness in Banach spaces, Studia Math. 11 (1950), 71-94.

[11] STUDEnÁ, A.: On Some Properties of Continuity of Vector Valued Functions. PhD Dissertation, Bratislava, 1985. (In Slovak) 


\title{
MILOSLAV DUCHOŇ — CAMILLE DEBIÈVE
}

[12] THOMAS, E.: The Lebesgue-Nikodym Theorem for Vector Valued Radon Measures. Providence, Rhode Island, 1974.

[13] TWEDDLE, I.: Weak compactness in locally convex spaces, Glasg. Math. J. 9 (1968), $123-127$.

Received January 5, 2011

\author{
Miloslav Duchon̆ \\ Mathematical Institute \\ Slovak Academy of Sciences \\ Štefánikova 49 \\ SK-814-73 Bratislava \\ SLOVAKIA \\ E-mail: miloslav.duchon@mat.savba.sk \\ Camille Debiève \\ Université Catholique de Louvain \\ Département de Mathématiques \\ 2, Chemin du Cyclotron \\ B-1348 Louvain-la-Neuve \\ BELGIUM \\ E-mail: camille.debieve@uclouvain.be
}

